\title{
ETIOLOGICAL PROFILE IN PATIENTS WITH VENTILATOR ASSOCIATED PNEUMONIA IN ICU AT A TERTIARY CARE HOSPITAL
}

Anil Mudda, Sanjeev Kumar. S. G, Ravi Kumar, Sai Shruthi, T. Anil Kumar

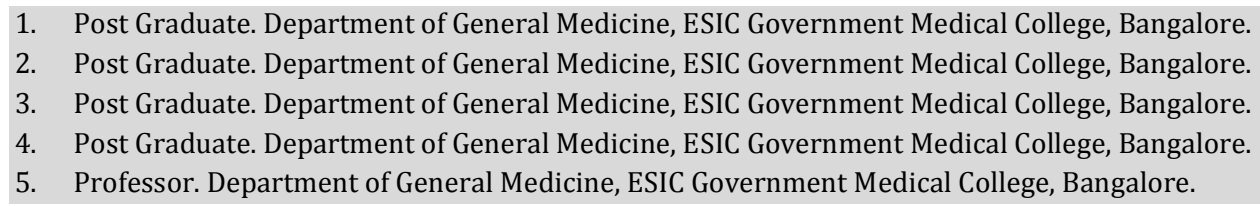

\section{CORRESPONDING AUTHOR:}

Dr. Anil Mudda,

Room no: f7, pg hostel

ESIC medical college,

Rajajinagar, Bangalore-10.

E-mail: anil_mudda@yahoo.com

ABSTRACT: AIM AND OBJECTIVES: The aim and objectives of the study is to know the etiological profile and infection rate of ventilator associated pneumonia(VAP) in ICU over one year period. MATERIALS AND METHODS: A hospital based study was carried out in the ICU in ESICMC \& PGIMSR Bangalore. This study was conducted from October 2011 to August 2012. The study was carried out amongst inpatients admitted to ICU and who were on ventilator.VAP was defined according to CDC criteria \&endotracheal aspirates were collected from eligible patients on ventilator. Identification of pathogens were performed as per guidelines. The data was analysed for determining VAP infection rate. RESULTS: A total of 173 patients were on ventilator in ICU. Among them 57 developed ventilator Associated pneumonia. VAP infection rate was $23 \%$. Acinetobacter baumannii\& Pseudomonas aeruginosa were commonest in VAP. The bacterial profile was found to be Acinetobacter baumannii 28 ( 49\% ),Pseudomonas aeruginosa 13 ( 23\% ),Klebsiella pneumonia 9( 16\% ), Escherichia coli 9 ( 16\% ) Staphylococcus aureus(MSSA) 2 ( $3.5 \%$ ) Enterobacter aerogenes 1 ( $1.75 \%$ ). CONCLUSION: The following conclusions were reached

1. VAP infection rate was $23 \%$.

2. A. baumannii \&Pseudomonas aeruginosa were predominant organisms.

KEY WORDS: VAP, endotracheal aspirates, VAP infection rate.

INTRODUCTION: Ventilator Associated Pneumonia (VAP) refers to a type of pneumonia that occurs more than 48-72 hours after endotracheal intubation, and is one of the most common nosocomial infections in patients receiving mechanical ventilation. ${ }^{1}$

Hospital Acquired Pneumonia (HAP), VAP, and Health Care Associated Pneumonia(HCAP) may be caused by a wide spectrum of bacterial pathogens, may be polymicrobial, and are rarely due to viral or fungal pathogens in immunocompetent hosts. ${ }^{2} \mathrm{VAP}$ is the commonest complication associated with Mechanical Ventilation(MV) reported at the rate of $1-3 \%$ per day of MV. Prevalence ranges from $10 \%$ to $65 \%$ in tertiary-care hospitals. ${ }^{3}$ VAP occurs approximately in $9-27 \%$ of all intubated patients. 4,5 Due to increasing incidence of multidrug-resistant (MDR) organisms in ICUs, early and correct diagnosis of VAP is an urgent challenge for optimal antibiotic treatment. Common pathogens include aerobic gram-negative bacilli, such as Pseudomonas aeruginosa, Escherichia coli, Klebsiella pneumoniae, and 
Acinetobacter species. Infections due to gram-positive cocci, such as Staphylococcus aureus , are more common in patients with diabetes mellitus and head trauma. ${ }^{6}$

Common pathogens include aerobic gram-negative bacilli, such as P. aeruginosa, Escherichia coli, Klebsiella pneumoniae, and Acinetobacter species. Infections due to grampositive cocci, such as Staphylococcus aureus, particularly methicillin resistant S. aureus (MRSA), have been rapidly emerging. Pneumonia due to $S$. aureus is more common in patients with diabetes mellitus, head trauma, and those hospitalized in ICUs.

Among the causes of hospital acquired pneumonias, Ventilator Associated Pneumonia (VAP) is important as it worsens the outcome and the cost of in-hospital treatment. VAP is a nosocomial pneumonia developing in a patient after 48 hours of mechanical ventilation, and could be early or late depending on the onset. The mortality and the morbidity associated with VAP depend on the early identification of the disease and the initiation of appropriate therapy. The use of appropriate antibiotics which are directed towards the most prevalent organism improves the cure rate and survival, and also reduces the emergence of resistant strains.

AIM OF THE STUDY: The aim of the study is to know the etiological profile and infection rate of ventilator associated pneumonia (VAP) in ICU.

Method of collection of data: Study design. A hospital based study was carried out in the ICU in ESICMC \&PGIMSR BANGALORE. : This study was conducted from October 2011 to August 2012. The study was carried out amongst inpatients admitted to ICU and who were on ventilator.

We assessed the clinical parameters (history and clinical examination) and investigations. This included the blood counts, renal function tests, blood glucose, liver function tests, electrocardiogram, endo-tracheal aspirates for gram staining and culture, blood culture, $\mathrm{ABG}$ and chest $\mathrm{x}$-rays or any other relevant investigations as and when required.

The clinical pulmonary infection score (CPIS) was tabulated from the available data includes temperature, leukocytes, tracheal aspirate volume and the purulence of tracheal secretions, chest X-ray, oxygenation- $\mathrm{PaO} 2 / \mathrm{FiO} 2$ and the semi-quantitative culture of the tracheal aspirates). The patients with CPIS which was more than 6, were considered to have developed VAP. VAP was diagnosed by the growth of pathogenic organisms.

INCLUSION CRITERIA: According to CDC definitions Ventilator Associated Pneumonia (VAP) : Adult patient on mechanical ventilation at the time of or within 48 hours before onset of the event and showing radiological evidence of pneumonia and Any 2 of the following:

1 . Temperature $\geq 38 \mathrm{C}$ or $\leq 35 \mathrm{C}$.

2. $\mathrm{WBC}>12000 / \mathrm{mm} 3$ or $<4000 / \mathrm{mm} 3$.

3. Purulent sputum.

4. Pathogenic bacteria isolated from endotracheal aspirate.

\section{EXCLUSION CRITERIA}

1. Patients having Pneumonia prior to MV

2. Patients intubated outside.

\section{DATA ANALYSIS:}

The data were analyzed by using the Chi square test. The level of significance was set at $\mathrm{P}<0.05$. 
RESULTS: A total of 440 patients were admitted in ICU over a period of one year. Among them 173 patients were on ventilator in ICU. In our study 57patients developed ventilator Associated pneumonia [Table 1].

VAP infection rate was $23 \%$. Acinetobacter baumannii\& Pseudomonas aeruginosa were commonest in VAP [Table 2].

The bacterial profile was found to be Acinetobacter baumannii 28 (49\%),Pseudomonas aeruginosa $13(23 \%)$, Klebsiella pneumonia 9 ( $16 \%)$, Escherichia coli 9 ( $16 \%$ ) Staphylococcus aureus(MSSA) 2 (3.5\% ) Enterobacter aerogenes ( $1.75 \%$ ).Fig 1.

DISCUSSION: Diagnosis and treatment of ventilator-associated pneumonia, a nosocomial infection that develops in mechanically ventilated patients and causes considerable morbidity and mortality, remain a challenge.7Pathogenic organisms causing VAP, along with complicating risk factors and comorbidities, result in extended hospitalization periods, escalated health care costs, and the requirement of potent, broad spectrum antimicrobial agents often used in expensive combination regimens. ${ }^{8}$

VAP is the commonest nosocomial infection amongst patients receiving MV in ICU. The incidence of VAP in our setting was 23\%.Table 3 and 4 shows the comparison of our study with other similar studies conducted with respect to incidence of VAP and etiological profile. Infection rates in studies conducted by Torres et alwas $24 \%$, Kerver et al 67\%,Kollef et al $15.5 \%$, Fagon et al 27.5\%,Rakshit et al 47\% respectively. ${ }^{9} \mathrm{VAP}$ infection rate in our study was $23 \%$.

The estimated prevalence of VAP ranges from 10 to $65 \%$, with a $20 \%$ case fatality .Ventilator-associated pneumonia is an important ICU infection in mechanically ventilated patients. It accounts for $13-18 \%$ of all hospital acquired infections. Data from recent studies shows that VAP was the most common infectious complication among patients who were admitted to the ICU. The complications and treatment cost significantly rises with VAP caused by resistant organisms, due to the cost of newer broad spectrum anti microbials and supportive measures. In various studies, the incidence of VAP was found to vary from $7 \%$ to $70 \%$. A similar incidence was found in studies done by Rakshit et al and Andrade et al. ${ }^{10,11}$ In our study it was $23 \%$.

The diagnostic criteria for VAP in patients receiving mechanical ventilation is the presence of two or more of the following clinical features: Temperature of $>38^{\circ} \mathrm{C}$ or $<36^{\circ} \mathrm{C}$; leukopenia or leukocytosis; purulent tracheal secretions and decreased $\mathrm{PaO2}$. If two or more of these abnormalities are present, a chest radiograph should be evaluated for alveolar infiltrates or an air bronchogram sign. Quantitative procedures for adequate sampling of the respiratory aspirates should be done, based on the local expertise and the cost considerations. Empirical anti microbial therapy and supportive care should be initiated by the subject's clinical state, clinical suspicion, and the available investigations.

The common pathogens which were isolated were the aerobic gram-negative bacilli such as Pseudomonas aeruginosa, Escherichia coli, Klebsiella pneumoniae and Acinetobacter species and gram-positive cocci like Staphylococcus aureus. Fungal pathogens are also not significant agents causing VAP.12,13

Recent studies have shown the increasing incidence of multidrug resistant pathogens (MDR) among the patients with VAP. A study by Dey et al showed the increased incidence of MDR pathogens in endo-tracheal aspirates. ${ }^{6}$ Earlier studies have shown that Pseudomonas was the most common organism. Staphylococcus was the most common organism in a study by Fagon et al, p. aeruginosa in a study by Torres et al and Rakshit et al. But in our study 
Acinetobacter found to be the most common organism causing VAP, followed by Pseudomonas species. Although the Acinetobacter species is less virulent than Pseudomonas, they are acquiring more and more resistance to the commonly used antimicrobial agents. Due to the increasing incidence of MDR organisms in ICUs, an early and correct diagnosis of VAP is a challenge for optimal antibiotic treatment. The emergence of MDR pathogens can be prevented by adopting an antibiotic institutional policy and dose de-escalation regimens .Isolation of the causative organism from ET secretions and its culture sensitivity is crucial in the management of VAP.

The early diagnosis and institution of appropriate antimicrobial therapy has shown reduced patient mortality. The mortality rates in VAP varied from $20-75 \%$, in different studies done by Rakshit et al and Andrade et al. 10,11

The incidence of VAP can be prevented by adopting careful intubation techniques, oral tuba tion, avoiding gastric over- distension, maintaining adequate endo tracheal cuff pressure and efficient tracheal toileting. Infection rate with MDR organisms are increasing in ICU set up. The mortality rate which was associated with VAP, was higher in patients aged above 60 years. The infection rates could possibly be reduced by practicing aseptic measures in the ICU. The overall outcome of VAPs could improve with the anti-microbial policies of individual centers.

\section{REFERENCES:}

1. Gupta A, Agrawal A, Mehrotra S, Singh A, Malik S, Khanna A. Incidence, risk stratification, antibiogram of pathogens isolated and clinical outcome of ventilator associated pneumonia. Indian J Crit Care Med 2011;15:96-101.

2. Am. J. Respir. Crit. Care Med. February 15, 2005 vol. 171 no. 4 388-416.

3. Rakshith P, Nagar V S, Deshpande A K. Incidence, clinical outcome and risk stratification of VAP- A prospective cohortstudy. Indian J of Crit Care Med. 2005; 9: 211-6

4. .Chastre J, Fagon JY. Ventilator-associated pneumonia. Am J RespirCrit Care Med 2002;165:867-903.

5. Rello J, Ollendorf DA, Oster G, Lionch MV, Bellm L, Redman R, et al . Epidemiology and outcomes of ventilator-associated pneumonia in a large US database. Chest 2002;122:2115-21.

6. .Dey A, Bairy I. Incidence of multidrug-resistant organisms causing ventilator-associated pneumonia in a tertiary care hospital: A nine months' prospective study. Ann Thorac Med 2007;2:52-7

7. Y. Fagon, J. Chastre, M. Wolff, C. Gervais, S. Parer-Aubas, F. Stéphan et al. "Invasive and Noninvasive Strategies for Management of Suspected Ventilator-Associated Pneumonia. A Randomized Trial".Ann Intern Med. 18 April 2000;132(8):621-630.

8. Ronald N. Jones Etiologies of HABP and VABP • CID 2010:51 (Suppl 1) • S81-87

9. Alp E and Voss A, Ventilator associated pneumonia and infection control, Annals of Clinical Microbiology and Antimicrobials. 2006, 5; 7: 1-11

10. Rakshith P, Nagar V S, Deshpande A K. Incidence, clinical outcome and risk stratification of VAP- A prospective cohort study. Indian J of Crit Care Med. 2005; 9: 211-13

11. Andrade L, Vilela C A P, Cezario R C, Almeida A B, Filho P G. Ventilator Associated Pneumonia in an Adult Clinical- Surgical Intensive Care Unit of a Brazilian University Hospital: Incidence, Risk Factors, Etiology, and Antibiotic Resistance. The Brazilian J InfDis. 2008; 12: 80-5. 
12. Krasinski K, Holzman RS, Hanna B, Greco MA, Graff M, Bhogal M. Nosocomial fungal infection during hospital renovation. Infect Control 1985;6:278-82. [PUBMED]

13. Ebiary M, Torres A, Fabregas N, Bellacasa JP, Gonzalez J, Ramirez J, et al . Significance of the isolation of Candida species from respiratory samples in critically ill, nonneutropenic patients. Am J RespirCrit Care Med 1997;156:583-90.

Table 1

\begin{tabular}{|l|l|l|l|}
\hline Months & $\begin{array}{l}\text { No of } \\
\text { patients } \\
\text { admitted } \\
\text { to ICU }\end{array}$ & $\begin{array}{l}\text { No of patients eligible for } \\
\text { study (on ventilator for } \\
\text { more than 48hrs) }\end{array}$ & $\begin{array}{l}\text { No of patients positive for } \\
\text { Ventilator } \\
\text { pneumonia(VAP) }\end{array}$ \\
\hline Octobsociated \\
\hline November & 35 & 14 & 6 \\
\hline December & 50 & 13 & 5 \\
\hline January & 42 & 15 & 6 \\
\hline February & 37 & 22 & 4 \\
\hline March & 36 & 15 & 4 \\
\hline April & 34 & 08 & 7 \\
\hline May & 49 & 18 & 3 \\
\hline June & 48 & 20 & 6 \\
\hline July & 29 & 13 & 7 \\
\hline August & 38 & 17 & 4 \\
\hline Total & 440 & 173 & 57 \\
\hline
\end{tabular}

Table 2

\begin{tabular}{|l|l|}
\hline Organisms & VAP ( \% ) \\
\hline Acinetobacter baumannii & $28(49 \%)$ \\
\hline Pseudomonas aeruginosa & $13(23 \%)$ \\
\hline Klebsiella pneumoniae & $9(16 \%)$ \\
\hline Escherichia coli & $4(7 \%)$ \\
\hline Staphylococcus aureus(MSSA) & $2(3.5 \%)$ \\
\hline Enterobacter aerogenes & $1(1.75 \%)$ \\
\hline Total & 57 \\
\hline
\end{tabular}


ORIGINAL ARTICLE

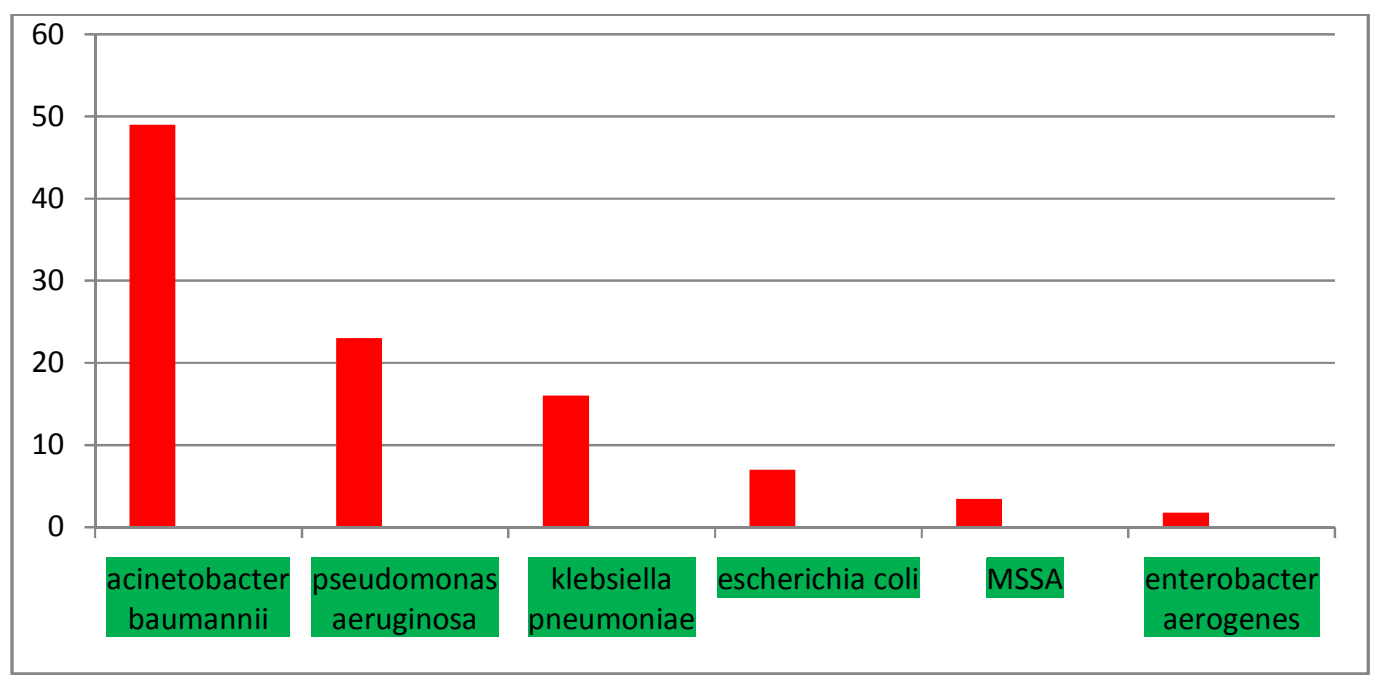

Figure 1

Table-3

\begin{tabular}{|l|l|l|}
\hline Authors No. of Patients Infection rate of VAP \\
\hline Kerver et al. & 39 & 67 \\
\hline Torres et al. & 322 & 24 \\
\hline Kollef et al. & 277 & 15.5 \\
\hline Fagon et al. & 1118 & 27.5 \\
\hline Rakshit.P et al & 51 & 47 \\
\hline Our study & 173 & 23 \\
\hline
\end{tabular}

Table 4

\begin{tabular}{|l|l|l|l|l|}
\hline \multicolumn{2}{|c|}{ Etiological profile of VAP(a comparison study) } \\
\hline Causative Organisms & Fagon et al., & Torres et al., & Rakshit et al., & our study \\
Pcinetobacter & $8(15 \%)$ & $6(24 \%)$ & $2(8 \%)$ & $28(49 \%)$ \\
Klebsiella & $16(31 \%)$ & $7(28 \%)$ & $11(46 \%)$ & $13(23 \%)$ \\
E. coli & $2(4 \%)$ & $3(12 \%)$ & $7(29 \%)$ & $9(16 \%)$ \\
Staphylococcus & $4(8 \%)$ & $3(12 \%)$ & $3(12 \%)$ & $4(7 \%)$ \\
& $17(33 \%)$ & $5(20 \%)$ & $6(25 \%)$ & $2(3.5 \%)$ \\
\hline
\end{tabular}

